\title{
Bayerische Private Akademie für Psychotherapie (BAP)
}

Die Bayerische Private Akademie für Psychotherapie BAP bietet wieder ab Herbst/Winter 1994 eine

verhaltenstherapeutische Zusatzausbildung in Kinder- und Jugendlichen-Therapie für Psychologen und Ärzte an.

Voraussetzung: Abgeschlossene VT-Ausbildung (oder Zwischenprüfung), Nachweis einer gleichwertigen Qualifikation bzw. Tätigkeit als verhaltenstherapeutischer Psychotherapeut im Abrechnungsverfahren.

Beginn: Herbst/Winter 1994 Dauer: etwa 1 Jahr

Die Ausbildung beínhaltet:

100 Stunden Theorie und fallzentrierte praktische Ausbildung (6 Wochenendworkshops)

5 eigene Kinder- oder Jugendlichenbehandlungen unter Supervision (diese können Bestandteil der 20 BAP-Fälle sein)

durchschnittlich jede 4. Therapiestunde eine Stunde Supervision

Die theoretische Ausbildung umfaßt folgende Themenkomplexe:

Entwicklungspsychologie, Verhaltensdiagnostik, Psychometrie, Entwicklungsdiagnostik, Gesprächsführungstechnik, Beobachtungsverfahren, Interventionsstrategien u.a.

die theoretische Ausbildung ist zeitlich gegliedert in Störungen im frühen Säuglings- und Kindesalter, Vorschul- und Grundschulalter, Jugendalter- und Familienstörungen.

Abschlußprüfung: Die Ausbildung schließt mit einem mündlichen

Kolloquium von 45 Minuten Dauer ab.Ausbildungsleitung: Dr. F. Peterander, Dr. Dr. S. Sulz Supervisoren und Dozenten: Dr. U. Brack, Dr. H. Dirlich-Wilhelm,

Dr. G. Görlitz, Dr. B. Hippler,

Dr. G. Leinhofer, Dr. F. Peterander,

Dr. Dr. S. Sulz, Dipl.-Psych. U. Streit

Kontaktadres.se:

BAP Gemeinnützige Gesellschaft mbH

Nymphenburger Straße 185

D. D-80634 München

Tel. (089)1684006 